\title{
Oxygen Control for an Industrial Pilot-scale Fed-batch Filamentous Fungal Fermentation
}

\author{
L. Bodizs ${ }^{1}$, M. Titica ${ }^{2, *}$, N. Faria ${ }^{3}$, \\ B. Srinivasan ${ }^{1, * *}$, D. Dochain ${ }^{2}$ and D. Bonvin ${ }^{1}$ \\ ${ }^{1}$ École Polytechnique Fédérale de Lausanne, \\ Lausanne, Switzerland \\ ${ }^{2}$ CESAME, Université Catholique de Louvain, \\ Louvain-la-Neuve, Belgium \\ ${ }^{3}$ Novozymes A/S, Bagsvaerd, Denmark
}

\begin{abstract}
Industrial filamentous fungal fermentations are typically operated in fed-batch mode. Oxygen control represents an important operational challenge due to the varying biomass concentration. In this study, oxygen control is implemented by manipulating the substrate feed rate, i.e. the rate of oxygen consumption. It turns out that the setpoint for dissolved oxygen represents a trade-off since a low dissolved oxygen value favors productivity but can also induce oxygen limitation. This paper addresses the regulation of dissolved oxygen using a cascade control scheme that incorporates auxiliary measurements to improve the control performance. The computation of an appropriate setpoint profile for dissolved oxygen is solved via process optimization. For that purpose, an existing morphologically structured model is extended to include the effects of both low levels of oxygen on growth and medium rheological properties on oxygen transfer. Experimental results obtained at the industrial pilot-scale level confirm the efficiency of the proposed control strategy but also illustrate the shortcomings of the process model at hand for optimizing the dissolved oxygen setpoints.
\end{abstract}

Key words: Bioprocesses, Filamentous fungal fermentation, Rheological properties, Oxygen control, Dissolved oxygen setpoint, Cascade control.

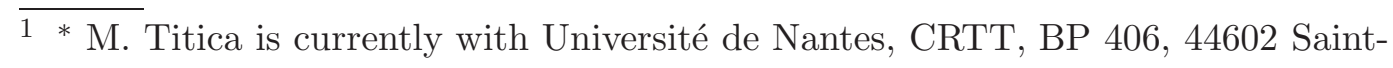
Nazaire, Cedex, France.

2 ** B. Srinivasan is currently with École Polytechnique de Montréal, Montréal, Canada. 


\section{Introduction}

Filamentous fungi are among the most frequently used cell factories in the fermentation industry. Their success is due to the relatively well-established fermentation technology and the versatility of strains available, thus allowing the production of a wide variety of products: primary metabolites, antibiotics, enzymes, and proteins [1].

Traditionally, filamentous fungal fermentations are operated in fed-batch mode, with the feed rates being manipulated manually. As a result of the filamentous structure of the biomass, its concentration is considerably higher than in other biological processes. High biomass concentration induces high viscosity, which makes oxygen transfer difficult [2]. In addition, other factors such as the initial properties of the biomass (inoculum) can lead to situations with insufficient oxygen and lower performance of the microorganisms. The goal of this work is to propose an oxygen control strategy that ensures high productivity and limits the risk of running into oxygen limitation.

Usually, the level of dissolved oxygen (DO) is controlled by manipulating the airflow or the stirrer speed. However, when applied to large-scale industrial reactors, which is the ultimate goal of this work, this strategy often leads to unacceptable power consumption. Additionally, this culture is rather shear sensitive because of the filamentous structure of the microorganisms. Hence, in these cases, the dissolved oxygen concentration can be controlled by limiting its consumption [3], [4], [5]. This is achieved by manipulating the substrate feed rate, i.e. the feed of the substance being oxidized. Both manual and PID control have been used for regulating the dissolved oxygen concentration, but the range in which this concentration could be confined is fairly wide. One way to cope with this difficulty is to operate with a sufficiently high setpoint for oxygen concentration so that, despite these large variations, oxygen limitation does not occur. However, this policy corresponds to feeding less substrate, which reduces production and is economically unacceptable. Hence, the objective is to control oxygen concentration tightly at a reasonably low setpoint so that oxygen limitation can be avoided without sacrificing production. Consequently, the objective of this project is twofold: i) optimization to determine a setpoint profile that maximizes production, and ii) control to follow this setpoint while avoiding oxygen limitation.

The first objective is addressed by solving a model-based optimization problem. A first-principles model of the filamentous fungal ( $\alpha$-amylase producing strain of Aspergillus oryzae) fermentation process is proposed in [6], while a data-driven model is described in [7]. Here, the former is utilized. This first-principles model is morphologically structured and provides a description of biomass, glucose and enzyme concentrations of submerged cultures of fil- 
amentous fungi without oxygen limitation. However, since oxygen dynamics are crucial in the operation of fed-batch cultivations, the influence of dissolved oxygen limitation on the biomass and enzyme production needs to be incorporated. These effects are included in the model, which is fitted and validated on experimental data from fed-batch fermentations provided by Novozymes, Denmark.

The control objective is met by a cascade-type controller. The poor performance of standard PID control can be attributed to the fact that it only uses information on the controlled variable, though much more information is available via auxiliary measurements such as the oxygen uptake rate $(O U R)$ and carbon dioxide evolution rate $(C E R)$. Hence, the idea to incorporate some of these measurements in the control algorithm. By analyzing the structure of the process model, a simple cascade control scheme is proposed, whereby the outer-loop oxygen controller provides the setpoint for the $O U R / C E R$ signal that is controlled in the inner loop.

Experimental results show that the setpoint chosen by model-based optimization is not appropriate for the real process. Though the model was fitted and validated on experimental data, its extrapolative power remains poor due to plant-model mismatch. However, the cascade controller is quite effective in keeping the dissolved oxygen in a tight range.

The paper is organized as follows. Section 2 describes the process under consideration and its operation in industry. Section 3 details a first-principles model of filamentous fungal fermentation and develops oxygen transfer relationships. Oxygen control is considered in Section 4, with optimization to determine the DO setpoint and cascade control for its regulatation. Section 5 presents experimental results carried out in a pilot plant at Novozymes. Finally, conclusions are provided in Section 6.

\section{Process Description and Industrial Practice}

\subsection{Fungal Fermentation}

The process studied in this paper is the $\alpha$-amylase production by Aspergillus oryzae. The same substrate is consumed for both biomass growth and enzyme production. The main difficulty with this process is oxygen limitation in the liquid phase. This depletion is usually caused by high biomass concentration, which, due to its filamentous structure, increases the viscosity and makes oxygen transfer difficult. 


\subsection{Current Operation}

The fermentation at Novozymes Pilot Plant, (Bagsvaerd, Denmark) is carried out in a $2500 \mathrm{~L}$ stirred vessel. $\mathrm{pH}$ is controlled through dosing ammonia $(\mathrm{g})$ and phosphoric acid. The fermenter is aerated at the constant rate of $1 \mathrm{vvm}$ and agitation speed of $275 \mathrm{rpm}$. Temperature and pressure are kept at a constant level by the process control system DeltaV, from Fisher Rosemount.

The typical way of operating the fermentation process is presented in Figure 1. For the sake of confidentiality, the experimental results have been normalized and thus no measurement units are presented. The substrate feeding policy takes into account the problem of oxygen limitation and consists of:

(1) a batch or growth phase, during which the substrate concentration is reduced from a high initial value to its operational range,

(2) a linearly-increasing feed rate whose role is to avoid oxygen limitation in the early phase of the fed-batch, and

(3) a 'constant' feed rate that is chosen in order to exactly fill the reactor in the remaining operation time and keep the dissolved oxygen around the desired value $D O_{\text {desired }}=25$.

As can be seen in Figure 1, the range of dissolved oxygen is fairly wide (20$40 \%$ ). Also, towards the end of the batch, increasing the feed rate causes oxygen limitation. A larger feed rate means more biomass, higher viscosity and thus reduced oxygen transfer. This phenomenon has been encountered and analyzed by the biologists in Novozymes. It can be explained as follows. Once under oxygen limitation, the biomass changes morphology, whereby the viscosity of the medium increases significantly. However, this increase in viscosity reduces oxygen transfer even more. This positive feedback effect leads to lower and lower oxygen levels and eventually to the death of the microorganisms. Hence, if tight oxygen control is not ensured, the risk of running into oxygen limitation is very high.

\subsection{Measurements}

The measurements available are the volume $V$, the viscosity $\eta$, the dissolved oxygen pressure $D O$, the aeration rate $Q_{g}$, the amount of oxygen consumed $\Delta\left[\mathrm{O}_{2}\right]$, and the amount of carbon dioxide produced $\Delta\left[\mathrm{CO}_{2}\right]$.

The volume is calculated from weight measurements, assuming constant density throughout the fermentation. The viscosity is determined by a viscosimeter Hydramotion, York England. DO is determined by an electrode Ingold, from Mettler Toledo. The oxygen and carbon dioxide concentrations in the 
exhaust gases are determined by a mass spectrometer VG Prima dB, from Thermo.

The oxygen uptake rate $O U R$ and the carbon dioxide evolution rate $C E R$ are determined from gas analysis. The inlet flow rate is measured, as are the mass fractions of oxygen and carbon dioxide in the inlet and the outlet flows. By using these quantities and a nitrogen (inert) balance, the volumetric output gas flow rate $G_{\text {out }}$ can be determined as follows:

$$
\begin{aligned}
& G_{\text {out }} y_{N_{2}, \text { out }}=G_{\text {in }} y_{N_{2}, \text { in }} \\
& G_{\text {out }}=G_{\text {in }} \frac{1-y_{O_{2}, \text { in }}-y_{C_{2}, \text { in }}}{1-y_{O_{2}, \text { out }}-y_{\mathrm{CO}_{2}, \text { out }}-y_{W, \text { out }}}
\end{aligned}
$$

where

$G_{\text {out }}$ - volumetric output gas flow rate $(\mathrm{nL} / \mathrm{h})$

$y_{N_{2}, \text { out }}$ - output mass fraction of nitrogen

$G_{i n}$ - volumetric input gas flow rate $(\mathrm{nL} / \mathrm{h})$

$y_{N_{2}, i n}$ - input mass fraction of nitrogen

$y_{O_{2}, \text { in }}$ - input mass fraction of oxygen

$y_{\mathrm{O}_{2} \text {,out }}$ - output mass fraction of oxygen

$y_{\mathrm{CO}_{2} \text {,out }}$ - output mass fraction of carbon dioxide

$y_{W, \text { out }}$ - output mass fraction of water

The output water mass fraction can be be calculated from the dilution of oxygen measurement, by purging the reactor without reaction [8]:

$$
y_{W, \text { out }}=y_{O_{2}, \text { in }}-y_{O_{2}, \text { out }}
$$

Once the output gas flow rate has been computed, OUR and CER can be determined from balance equations without accumulation terms:

$$
\begin{aligned}
& O U R=\frac{G_{\text {in }} y_{\mathrm{O}_{2}, \text { in }}-G_{\text {out }} y_{\mathrm{O}_{2}, \text { out }}}{V} \frac{\rho}{M_{\mathrm{O}_{2}}} \\
& C E R=\frac{G_{\text {out }} y_{\mathrm{CO}_{2}, \text { out }}-G_{\text {in }} y_{\mathrm{CO}_{2}, \text { in }}}{V} \frac{\rho}{M_{\mathrm{CO}_{2}}}
\end{aligned}
$$


where $\rho$ is the average density of the gas flow, while $M_{\mathrm{O}_{2}}$ and $M_{\mathrm{CO}_{2}}$ are the molecular weights for oxygen and carbon dioxide, respectively.

Note that, in general, an acceptable approximation is to simply consider $O U R \approx O T R$, since the solubility of oxygen is very low [9]. Typically, the same does not hold for $C E R$ and $C T R$ since the solubility of carbon dioxide depends on the physical and chemical properties of the medium, such as temperature and $\mathrm{pH}$. However, since here the fermentor is operated at constant temperature, pressure and $\mathrm{pH}$ in the fed-batch phase (Figure 1), it can be assumed that the rate at which $\mathrm{CO}_{2}$ is formed by microbial metabolism corresponds to the carbon dioxide transfer rate and thus $C E R \approx C T R$.

\section{$3 \quad$ Filamentous Fungal Fermentation Model}

In this section, the influence of dissolved oxygen is incorporated within the kinetic equations using Monod expressions, and an additional state - for dissolved oxygen - is introduced in the model.

\subsection{Morphology and Rate Expressions}

Growth kinetics. The morphologically structured model proposed by [6] is based on the division of the biomass into three different compartments:

- Active region $\left(X_{a}\right)$ - responsible for the uptake of substrate and growth of the hyphal elements. It is assumed that only the active region is responsible for enzyme production.

- Extension region $\left(X_{e}\right)$ - responsible for new cell wall generation and extension.

- Hyphal region $\left(X_{h}\right)$ - the degenerated part of the hyphal elements that is inactive.

The macroscopic reactions for growth and production can be expressed as:

$$
\begin{aligned}
& S+O_{2} \stackrel{X_{a}}{\rightarrow} X_{e} \\
& S+O_{2} \stackrel{X_{e}}{\longrightarrow} X_{a} \\
& X_{a} \rightarrow X_{h} \\
& S+O_{2} \stackrel{X_{a}}{\longrightarrow} P
\end{aligned}
$$


where $S$ stands for the substrate (glucose), $\mathrm{O}_{2}$ for the dissolved oxygen and $P$ for the product ( $\alpha$-amylase).

The corresponding kinetic expressions read:

Branching (Equation (6)):

$$
q_{1}=x_{a} \frac{D O}{D O+K_{D O}} \frac{k_{1} s}{a_{t}\left(s+K_{s 1}\right)}
$$

Growth of the active region (Equation (7)):

$$
q_{2}=a_{t} x_{e} \frac{D O}{D O+K_{D O}} \frac{k_{2} s}{s+K_{s 2}}
$$

Differentiation (Equation (8)):

$$
q_{3}=k_{3} x_{a}
$$

The specific growth rate of total biomass is:

$$
\mu=\frac{q_{2}}{x_{t}}
$$

where $x_{t}=x_{e}+x_{a}+x_{h}$ represents the total biomass concentration, $x_{e}, x_{a}$ and $x_{h}$ are the concentrations of the extension, active and hyphal zones, respectively, $s$ and $D O$ are the substrate and dissolved oxygen concentrations, $a_{t}$ represents the number of tips per unit mass of the extension zones. The parameter $a_{t}$ is described as a function of $\mu$ (see $[6,10]$ for details concerning the morphological model). The kinetic expressions and the model parameters are presented in Appendix A.

Specific rate of enzyme production. Enzyme production in filamentous fungi is a classical example of growth-associated product formation. The enzyme production is subject to glucose (substrate) inhibition and oxygen limitation:

$$
r_{p s}=\left(\frac{\mu_{0} s}{K_{s}+s+\frac{s^{2}}{K_{I}}}+k_{c} \frac{s}{s+K_{c o r}}\right) \frac{D O}{D O+K_{D O}}
$$


The expression used in [6] describes the substrate inhibition by an exponential decrease in the specific production rate of the enzyme when the substrate concentration exceeds a certain threshold value. In this study, the expression of the specific rate of enzyme production is modified by using a Haldane expression to describe the substrate inhibition. This modification has been motivated by the application of an extremum-seeking controller to this process [11]. The parameter $k_{c}$ quantifies the constitutive level of enzyme production at high glucose concentrations (during the batch phase).

The specific rate of dissolved oxygen consumption is expressed as:

$$
r_{D O}=Y_{X O} \frac{q_{2}}{x_{t}}+Y_{P O} r_{p s} \frac{x_{a}}{x_{t}}+m_{o} \frac{D O}{D O+K_{D O}}
$$

where $Y_{X O}$ and $Y_{P O}$ are the yields of dissolved oxygen consumption for growth and enzyme production, respectively, and $m_{o}$ is the maintenance coefficient that represents the oxygen consumption of biomass.

The specific rate of substrate consumption is expressed as:

$$
r_{s}=Y_{X S} \frac{q_{2}}{x_{t}}+Y_{P S} r_{p s} \frac{x_{a}}{x_{t}}+m_{s} \frac{D O}{D O+K_{D O}}
$$

where $Y_{X S}$ and $Y_{P S}$ are the yields of substrate consumption for growth and enzyme production, respectively, and $m_{s}$ is the maintenance coefficient (based on the total amount of biomass).

\subsection{Mass Balance Equations}

The model proposed in [6] consists of a set of five balance equations for the three regions of biomass, the substrate and the product concentrations, as given in equations (17) - (19). An additional mass balance is included here to describe the dissolved oxygen concentration in the bioreactor (20). Furthermore, eq. (21) describes the bioreactor volume that varies with time and determines the end of the fermentation process.

Morphological states $x_{e}, x_{a}$ and $x_{h}$ 


$$
\begin{aligned}
& \dot{x}_{e}=q_{1}-\frac{F}{V} x_{e}, \quad x_{e}(0)=x_{e 0} \\
& \dot{x}_{a}=q_{2}-q_{1}-q_{3}-\frac{F}{V} x_{a}, \quad x_{a}(0)=x_{a 0} \\
& \dot{x}_{h}=q_{3}-\frac{F}{V} x_{h}, \quad x_{h}(0)=x_{h 0}
\end{aligned}
$$

Glucose s

$$
\dot{s}=-r_{s} x_{t}+\frac{F}{V}\left(s_{f}-s\right), \quad s(0)=s_{0}
$$

Enzyme p

$$
\dot{p}=r_{p s} x_{a}-\frac{F}{V} p, \quad p(0)=p_{0}
$$

Dissolved oxygen DO

$$
\dot{D O}=-r_{D O} x_{t}+k_{L} a\left(D O^{*}-D O\right)-\frac{F}{V} D O, \quad D O(0)=D O_{0}
$$

Volume $V$

$$
\dot{V}=F-F_{\text {evap }}, \quad V(0)=V_{0}
$$

In these equations, $F$ represents the substrate feeding rate, $s_{f}$ is the substrate concentration in the feed, $F_{\text {evap }}$ is the evaporation rate, $k_{L} a$ the specific gasliquid mass transfer coefficient for oxygen, and $D O^{*}$ the oxygen saturation concentration.

The model parameters and their numerical values are given in Appendix A. The parameters related to the microscopic morphology (10)-(11) are taken from [6]. The kinetic parameters for the Haldane expression (14) are identified by fitting the model to simulated data generated by the model in [6]. The yield coefficients $Y_{X S}, Y_{P S}, Y_{X O}, Y_{P O}$ are identified from experimental data provided by Novozymes. The data used for model fitting include both on-line measurements (DO, viscosity, $O U R, C E R$, and $V$ ) and off-line measurements $\left(p\right.$ and $\left.x_{t}\right)$. 


\subsection{Modeling Oxygen Transfer}

The effect of oxygen on growth and production is crucial due to the positive feedback effect mentioned in Section 2.2. Hence, it is necessary to understand and model the factors that affect oxygen transfer. This will be done via an empirical model that relates $k_{L} a$ to the state variables $x_{t}$ and $D O$. This link will be established through the viscosity and consists of various steps:

(1) Estimation of $k_{L} a$. In stirred bioreactors, the oxygen transfer coefficient, $k_{L} a$, depends on the impeller characteristics and the rheological properties of the fermentation medium. The value of $k_{L} a$ can be determined experimentally by the dynamic OTR method (oxygen transfer rate measurement) that gives an average $k_{L} a$ value for the bioreactor [12]. Online measurements of oxygen transfer rate (OTR), viscosity, and $D O$ are available, as well as off-line measurement of the total biomass $x_{t}$. The $k_{L} a$-value is calculated as:

$$
k_{L} a=\gamma \frac{O T R}{\left(D O^{*}-D O\right)}
$$

where $\gamma$ is a proportionality factor.

(2) Calibration of $k_{L}$ a as a function of viscosity. An empirical relationship between $k_{L} a$ and viscosity is determined experimentally. Under maximum air flow rate and constant agitation speed, the substrate feed rate is varied in such a way that oxygen limitation occurs. $k_{L} a$ is estimated as mentioned above and viscosity is measured. The relationship can be approximated by the linear equation (Figure 2):

$$
k_{L} a=c_{0}-c_{1} \eta
$$

where $\eta$ represents the on-line measurement of viscosity, and $c_{0}$ and $c_{1}$ are linear regression coefficients $\left(R^{2}=0.94\right)$.

(3) Calibration of viscosity as a function of $x_{t}$ and DO. The use of eq. (23) in the dynamic model (17) - (21) requires knowledge of viscosity. For a given stirrer speed, the viscosity of the filamentous suspension is influenced by two main factors: the biomass concentration and the fibrous structure of the biomass. Due to the lack of quantification of the fibrous structure, the viscosity is simply regressed with available experimental data. A linear relationship between viscosity and biomass concentration is adequate to represent experimental observations (data not shown). This is also in agreement with literature data for fermentations of Penicillium chrysogenum and Aspergillus niger [12]. Nevertheless, this relation can be improved by introducing the effect of dissolved oxygen to give:

$$
\eta=\eta_{0}+a_{X} x_{t}-a_{D O} D O
$$


where $\eta_{0}, a_{X}$, and $a_{D O}$ are linear regression coefficients $\left(R^{2}=0.84\right)$. A negative effect of $D O$ on the viscosity is found. This effect can be explained intuitively by the behavior of the filamentous organisms considered in this paper. Following a decrease in the $D O$ level, the microorganisms react by 'reaching out for air', that is changing their fibrous structure so as to increase the zone of the reaction mass from where they can absorb oxygen. As a consequence, viscosity increases (see Section 2.2). Also, when the level of $D O$ is high, there is enough 'air to breathe' within the immediate neighborhood, so there is no need to stretch any further, thereby keeping viscosity low. The performance of the viscosity model is shown in Figure 3.

In summary, by combining equations (23) and (24), it is possible to model the oxygen transfer coefficient $k_{L} a$ in terms of total biomass and dissolved oxygen. The accuracy of the $D O$ prediction using equation (20) to model the dynamics of $D O$ and equations (23) and (24) to express $k_{L} a$ in terms of $x_{t}$ and $D O$ is presented in Figures 4 and 5 for two different data sets. It is seen that the oxygen model performs satisfactorily.

The simulated behavior of the reactor is presented in Figure 6. It can be observed that the substrate concentration is much higher at the beginning, in the batch phase, than later on, in the fed-batch phase. This strategy favors biomass growth in the batch phase and production in the fed-batch phase. It is also observed that, in the fed-batch phase, the amount of inactive biomass $X_{h}$ increases. As a consequence, the viscosity of the medium increases, leading to a decrease of the oxygen transfer rate ( $k_{L} a$ decreases). This decrease, in turn, reduces the dissolved oxygen concentration, which might lead to oxygen limitation.

\section{Oxygen Control}

\subsection{Setpoint Computation for Dissolved Oxygen}

The model given in the previous section can be used to determine the dissolved oxygen profile that would maximize the bioreactor performance while avoiding oxygen limitation. This optimization problem can be formulated mathematically as follows [13]: 


$$
\begin{aligned}
\min _{F(t), t_{f}} & t_{f} \\
\text { s.t. } & (10)-(21),(23),(24) \\
& p\left(t_{f}\right) V\left(t_{f}\right) \geq\left[p\left(t_{f}\right) V\left(t_{f}\right)\right]_{\min } \\
& V\left(t_{f}\right) \leq V_{\max } \\
& F_{\min } \leq F(t) \leq F_{\max }
\end{aligned}
$$

where $t_{f}$ is the final time, $\left[p\left(t_{f}\right) V\left(t_{f}\right)\right]_{\min }$ is the minimum amount of enzyme to be produced, $V_{\max }$ is the maximum volume, $F_{\min }$ and $F_{\max }$ are the minimum and maximum feed rates, respectively.

Note that this optimization can be performed once before the beginning of the batch or repeated several times during the batch. If the optimization is repeated, the available measurements can be used in the optimization via a software sensor that estimates the current states (Figure 7). In this study, a software sensor based on open-loop model prediction is used [14]. This software sensor is based on the model used for optimization, except that the equations corresponding to the measured states $D O$ and $V$ are discarded since the state values are known from measurements. Furthermore, $k_{L} a$ is computed as in equation (22).

Usually, the goal of such an optimization is to compute the control inputs directly [15]. In this study, however, not the feed rate profile $F(t)$ but the dissolved oxygen profile $D O(t)$ is sought. This profile is used as reference profile for the cascade controller to be discussed in the next subsection. The strategy is illustrated in Figure 7.

\subsection{Cascade Control}

A cascade control structure is chosen since it has been observed experimentally at Novozymes that simple output controllers for $D O$ control do not perform well. Hence, additional measurements are sought to improve the control performance. The auxiliary measurements that are available for this process are $p H$, pressure, viscosity, $V, O U R$ and $C E R$. Since $p H$ and pressure have not been incorporated in the model and the viscosity sensor is not always reliable, the idea is to develop a control strategy that uses the $O U R, C E R$ and $V$ measurements.

An interesting feature can be taken from the oxygen dynamics. The feed rate has two main effects on the dissolved oxygen concentration: (i) an immediate effect through dilution (the last term on the right-hand side of eq. (20)) since increasing $F$ decreases $D O$, and (ii) integral effect through oxygen consumption (first term) since increasing $F$ increases the amount of substrate in 
the reactor and thus also its consumption, which in turn decreases $D O$. Furthermore, the influence of $F$ on the oxygen transfer between the gaseous and the liquid phases (second term) is quite negligible compared to the other two terms.

If the $D O$ level is controlled through dilution only (the last term on the righthand side of eq. (20)), the resulting change in the amount of substrate may itself induce a change in $D O$, which gives rise to oscillations and poor performance. This is typical of systems where the zero dynamics play an important role in process performance [16].

This problem can be better understood by considering a simplified linearized version of Problem (17)-(21) with both a direct and an integral effect of $u$ : $\dot{x}_{1}=a x_{2}+u$ and $\dot{x}_{2}=u$, where $a \geqslant 0$ is a coefficient that represents the relative importance of the integral effect (Figure 8). With the input $u$ and the controlled output $x_{1}$ the transfer function reads $\frac{X_{1}(s)}{U(s)}=\frac{s+a}{s^{2}}$. Using the proportional controller $k$ shown in Figure 9 , the closed-loop characteristic polynomial becomes $s^{2}+k s+a k$, which will have real roots for $k \geqslant 4 a$. Hence, in the presence of a fast zero (a large), one needs to use a very large controller gain to ensure non-oscillatory behavior. Such a large gain may not be admissible in practice due to model mismatch and measurement errors.

An efficient way of controlling this second-order system is to use the cascade scheme given in Figure 10, i.e. $u=k_{i}\left[k_{o}\left(x_{1, s p}-x_{1}\right)-x_{2}\right]$. Note that this cascade scheme corresponds to full-state feedback for this second-order system. The characteristic polynomial is $s^{2}+k_{i}\left(k_{o}+1\right) s+a k_{i} k_{o}$, the poles of which can be placed anywhere independently of the value of a.

Hence, the proposed cascade scheme is one way of implementing state feedback for the part of the dynamics that concerns dissolved oxygen.

The term $r_{D O} x_{t}$ can be evaluated via $O U R$ and $C E R$ measurements. This can be understood from eq. (15) that shows that $r_{D O}$ is the total oxygen consumptions for growth, production and maintenance. This oxygen consumption is approximately reflected by the $O U R$ measurement. Furthermore, the $C E R$ measurement is an indication of biomass activity and its concentration in the medium. For the operating conditions used in this experiment (constant $\mathrm{CO}_{2^{-}}$ solubility), OUR and CER are both directly related to $r_{D O} x_{t}$. Hence, $r_{D O} x_{t}$ can be approximated as $\beta \frac{O U R+C E R}{V}$, where $\beta$ is a proportionality factor. Note that a controller using only OUR measurements would probably work as well. Unfortunately, such a controller was not tested as part of this work.

The idea of the cascade control is to use $\left(r_{D O} x_{t}\right)_{s p}$ as the manipulated variable to control $D O$, while $F$ is used to control $r_{D O} x_{t}, x_{t}$ being a function of $F$ as shown by eq. (17). This leads to the cascade structure of Figure 11, in which 
the role of the inner loop is to regulate the substrate consumption based on $O U R$ and $C E R$ measurements, while the outer loop gives the setpoint for this consumption.

\subsection{Controller Tuning}

Since, for the system at hand, cascade control corresponds to state feedback, there are sufficient degrees of freedom to place the poles of the closed-loop system arbitrarily in the complex plane. Hence, the performance of the closed-loop system (including its stability) is determined by placing the poles appropriately in the complex plane. Ideally, the desired poles are determined from some theoretical considerations, as explained in standard textbooks [17]. For this, however, the nonlinear process model has to be linearized and the controller gains determined on the basis of the linear approximation.

In this study, controller tuning was carried out directly on the nonlinear process model by trial and error. The advantage of this approach is that it avoids the errors induced by linearization, while having the disadvantage of being empirical. Nevertheless, the controller tuning was successful, as shown in the next section.

Furthermore, a PID controller was also tuned empirically. However, due to the presence of a zero in the oxygen dynamics, as pointed out in the previous section, a PID controller does not have sufficient degrees of freedom for arbitrary pole placement, thereby resulting in less satisfactory results. Note, however, that PID control may, in some cases, provide good regulatory performance; for example, a system without zero is handled successfully by PI control in $[5]$.

\section{Experimental Results}

The performance of PID control to regulate the process around a constant setpoint $\left(D O_{s p}=25\right)$ is illustrated in the second phase of Experiment II (Figure 12). The performance is rather poor, with a large DO variability $(25 \pm 10)$. In contrast, the cascade controller used in the third phase of the experiment is able to keep the $D O$ concentration within the interval $25 \pm 2$, which is a major improvement to both manual operation and standard PID control.

The cascade controller is further tested in Experiment III (Figure 13). The setpoint for this experiment has been obtained by solving the optimization problem (25) off-line before the beginning of the batch, i.e. without the soft- 
ware sensor given in Figure 7. Since the resulting DO-profile is nearly constant, it is approximated by a constant value for ease of implementation [13]. The controller works satisfactorily for most of the time. However, the oscillatory behavior typical of oxygen limitation starts after 0.75 time units. Since this phenomenon of oxygen limitation is highly nonlinear, the linear cascade controller is unable to bring the fermentation back to normal operation.

The on-line reoptimized $D O$ setpoint given in Figure 7 is used in the second phase of Experiment IV (Figure 14). Unfortunately, the optimization provides setpoints that are well below $D O_{s p}=20 \%$, already known from Experiment III to lead to oxygen limitation. Since this is considered unacceptable, a safe and constant setpoint is used in the third phase of this experiment. In this third phase, several external perturbations act on the system, ranging from sensor failure at time 0.55 to manual setpoint changes at time 0.7 .

These experiments have demonstrated that, even though the model has been fitted to give good one-step ahead prediction and fairly reasonable multi-step simulations, the setpoints provided by solving optimization problem (25) offline (Experiment III) or on-line (Experiment IV) are in the region of oxygen limitation. This observation can be explained by the plant-model mismatch present in the oxygen dynamics. However, these experiments have shown that the cascade controller is able to follow the desired DO setpoint more accurately than traditional PID control.

Unfortunately, though the regulatory performance of the controller is excellent, the transients exhibit some overshoot. This is mainly due to input saturation not being accounted for in the control design. Other strategies, such as controlling $O U R$, could be applied since they give a more reliable indication of biomass activity than $D O$ early in the batch.

\section{Conclusions}

The main outcome of this study is the cascade controller for dissolved oxygen. The proposed scheme efficiently regulates the $D O$ level, but a proper setpoint needs to be chosen to keep the process away from oxygen limitation. The advantage of the proposed control strategy is that it is simple to apply and not specific to the strain of microorganism studied in this paper. Thus, it is applicable to a wide variety of fungal fermentations.

A mechanistic model of enzyme production by filamentous fungal fermentation has been extended to include rheological considerations. The effect of medium viscosity on the oxygen dynamics is described by empirically modeling the dependence of the mass transfer coefficient $k_{L} a$ on viscosity and that of vis- 
cosity on biomass and dissolved oxygen concentration. The resulting model is used to determine the optimal $D O$ setpoint, while the insight gained from the model is also used to design a cascade controller. This study has also indicated several model shortcomings. The most important one is that the effect of $\mathrm{pH}$ is completely discarded. As a consequence, the model predicts feasible operation at low dissolved oxygen values that could not be verified experimentally. Also, the model-based optimization is not capable of computing reliable DO setpoints.

In future experiments, one could focus on determining experimentally the best setpoint for dissolved oxygen, e.g. using extremum-seeking techniques [11].

Acknowledgements: This paper presents the results of the Knowledge-driven Batch Production (BatchPro) European Project HPRN-CT-2000-00039. The authors would like to thank F. Lei and H. Jorgensen from Novozymes, Bagsvaerd, Denmark, for their suggestions and for making possible to carry out the experimental work.

\section{References}

[1] M. McIntyre, C. Muller, J. Dynesen, J. Nielsen, Metabolic engineering of the morphology of Aspergillus, Adv. Biochem. Eng./Biotech. 73 (2001) 104-128.

[2] Z. J. Li, V. Shukla, A. P. Fordyce, A. G. Pedersen, K. S. Wenger, M. R. Marten, Fungal morphology and fragmentation behavior in fed-batch Aspergillus oryzae fermentation at the production scale, Biotech. Bioeng. 70 (2000) 300-312.

[3] R. Oliveira, R. Simutis, S. F. de Azevedo, Design of a stable adaptive controller for driving aerobic fermentation processes near maximum oxygen transfer capacity, J. Process Contr. 14 (2004) 617-626.

[4] L. Simon, M. N. Karim, Identification and control of dissolved oxygen in hybridoma cell culture in a shear sensitive environment, Biotechnol. Prog. 17 (2001) 634-642.

[5] J. D. Chung, Design of metabolic feed controllers: Application to high-density fermentations of Pichia pastoris, Biotech. Bioeng. 68 (2000) 298-307.

[6] T. Agger, B. Spohr, M. Carlsen, J. Nielsen, Growth and product formation of aspergillus oryzae during submerged cultivations: Verification of a morphologically structured model using fluorescent probes, Biotech. Bioeng. 57 (1998) 321-329.

[7] J. K. Rasmussen, S. B. Jorgensen, Experimental investigation of data-driven modelling for control of fed-batch cultivation, in: IFAC World Congress, Prague, Czech Republic, 2005. 
[8] C. Herwig, On-line exploitation tools for the quantitative analysis of metabolic regulations in microbial cultures, Ph.D. thesis, Ecole Polytechnique Federale de Lausanne (2001).

[9] A. G. Pedersen, $k_{L} a$ characterization of industrial fermentors, in: $4^{\text {th }}$ International Conference on Bioreactor and Bioprocess Fluid Dynamics, Edinburgh, UK, 1997.

[10] L. Bodizs, B. Srinivasan, D. Bonvin, Optimal feeding strategy for bioreactors with biomass death, in: Proc. CAB9, Nancy, France, 2004.

[11] M. Titica, D. Dochain, M. Guay, Adaptive extremum-seeking control of enzyme production in filamentous fungal fermentation, in: Proc. CAB9, Nancy, France, 2004 .

[12] A. Moser, Bioprocess Technology. Kinetics and Reactors., Springer-Verlag, 1988.

[13] M. Titica, L. Bodizs, F. Lei, B. Srinivasan, D. Dochain, D. Bonvin, Modeling and optimization of fed-batch filamentous fungal fermentation, in: BatchPro Symposium, Poros, Greece, 2004, pp. 55-65.

[14] D. Dochain, State and parameter estimation in chemical and biochemical processes : A tutorial, J. Process Contr. 13 (2003) 801-818.

[15] B. Srinivasan, S. Palanki, D. Bonvin, Dynamic optimization of batch processes: I. Characterization of the nominal solution, Comp. Chem. Eng. 27 (2003) 1-26.

[16] P. Mullhaupt, B. Srinivasan, J. Levine, D. Bonvin, Cascade control of the toycopter, in: ECC'99, Karlsruhe, Germany, 1999.

[17] D. E. Seborg, T. F. Edgar, D. A. Mellichamp, Process Dynamics and Control, Wiley, 1989. 
A Kinetic Expressions and Model Parameters for the Fed-batch Filamentous Fermentation Model

$$
\begin{aligned}
& k_{1}=\frac{k_{\text {bran }} \cdot 10^{4}}{\frac{\pi}{4}\left(d \cdot 10^{-4}\right)^{2}(1-w) f \rho} \\
& a_{t}=\left(\frac{1}{2}\left(\frac{1}{2} d \cdot 10^{-4}\right)^{3} \frac{4 \pi}{3}(1-w) \rho\right)^{-1} \\
& k_{2}=k_{\text {tip, max }} \cdot 10^{-4} \frac{\pi}{4}\left(d \cdot 10^{-4}\right)^{2}(1-w) f \rho \\
& d=\frac{1.1+\sqrt{1.21+\frac{135 k_{t i p, \text { max }} f s x_{e}}{\left(s+K_{s 2}\right)\left(x_{e}+x_{a}+x_{h}\right)}}}{2}
\end{aligned}
$$


Table A.1

Model parameter values

\begin{tabular}{|c|c|c|}
\hline Parameter & Value & Measurement unit \\
\hline$a_{D O}$ & 0.04 & $\mathrm{~kg} /(\mathrm{m} \mathrm{s} \%)$ \\
\hline$a_{x}$ & 0.094 & $\mathrm{~kg}$ DW kg/(g m s) \\
\hline$\beta$ & 1 & $\mathrm{~L} / \mathrm{mmol}$ \\
\hline$\gamma$ & 1 & $1 / \mathrm{mmol}$ \\
\hline$c_{0}$ & 67.2 & $h^{-1}$ \\
\hline$c_{1}$ & 4.3816 & $\mathrm{~m} \mathrm{~s} /(\mathrm{kg} \mathrm{h})$ \\
\hline$D O^{*}$ & 100 & $\%$ \\
\hline$\eta_{0}$ & 4.185 & $\mathrm{~kg} /(\mathrm{m} \mathrm{s})$ \\
\hline$f$ & 80 & $\%$ \\
\hline$F_{\text {evap }}$ & 1.25 & $\mathrm{~L} / \mathrm{h}$ \\
\hline$k_{3}$ & 0.08 & $h^{-1}$ \\
\hline$k_{\text {bran }}$ & 0.0017 & $\operatorname{tip} /(\mu \mathrm{m} \mathrm{h})$ \\
\hline$k_{c}$ & 8 & FAU kg DW/(L g h) \\
\hline$K_{\text {cor }}$ & $10^{-6}$ & $\mathrm{~g} / \mathrm{L}$ \\
\hline$K_{I}$ & $1.5 \cdot 10^{-3}$ & g glucose/L \\
\hline$K_{D O}$ & 2.5 & $\%$ \\
\hline$K_{S}$ & 0.0211 & g glucose /L \\
\hline$K_{s 1}$ & 0.003 & g glucose /L \\
\hline$K_{s 2}$ & 0.006 & g glucose /L \\
\hline$k_{t i p, \max }$ & 49 & $\mu \mathrm{m} /(\operatorname{tip} h)$ \\
\hline$m_{o}$ & 0.01 & $\% \mathrm{~kg} \mathrm{DW} /(\mathrm{g} h)$ \\
\hline$m_{s}$ & 0.01 & $\mathrm{~kg}$ DW g glucose $/(\mathrm{g} \mathrm{L} h)$ \\
\hline$\mu_{0}$ & 227 & FAU $\mathrm{kg}$ DW $/(\mathrm{L} g h)$ \\
\hline$\rho$ & 1 & $\mathrm{~g} / \mathrm{cm}^{3}$ \\
\hline$s_{f}$ & 430 & g glucose / L \\
\hline$w$ & 0.67 & $\mathrm{~g} / \mathrm{kg} \mathrm{DW}$ \\
\hline$Y_{X S}$ & 1.75 & g glucose kg DW / L g \\
\hline$Y_{P S}$ & $1.88 \cdot 10^{-4}$ & $\mathrm{~g}$ glucose / FAU \\
\hline$Y_{X O}$ & 57 & $\% \mathrm{~kg} \mathrm{DW} / \mathrm{g}$ \\
\hline$Y_{P O}$ & 35 & $\% \mathrm{~L} / \mathrm{FAU}$ \\
\hline
\end{tabular}




\section{B Variables used in the Fed-batch Filamentous Fermentation Model}

$a_{t}$ - number of tips per unit mass (tips/(kg DW))

$a_{D O}$ - regression coefficient $(\mathrm{kg} /(\mathrm{m} \mathrm{s} \%))$

$a_{x}$ - regression coefficient $(\mathrm{kg} \mathrm{DW} \mathrm{kg} /(\mathrm{g} \mathrm{m} \mathrm{s}))$

$\beta$ - proportionality coefficient $(\mathrm{L} / \mathrm{mmol})$

$\gamma$ - proportionality coefficient $(1 / \mathrm{mmol})$

$C E R$ - Carbon Evolution Rate $(\mathrm{mmol} /(\mathrm{L} \mathrm{h}))$

$c_{0}$ - regression coefficient $\left(h^{-1}\right)$

$c_{1}$ - regression coefficient $(\mathrm{m} \mathrm{s} /(\mathrm{kg} \mathrm{h}))$

$d$ - hyphal diameter $(\mu \mathrm{m})$

$\Delta\left[\mathrm{CO}_{2}\right]$ - change in carbon dioxide concentration over the reactor (\%)

$\Delta\left[\mathrm{O}_{2}\right]$ - change in oxygen concentration over the reactor (\%)

$D O$ - dissolved oxygen concentration (\%)

$D O^{*}$ - equilibrium dissolved oxygen concentration (\%)

$\eta$ - viscosity $(\mathrm{kg} /(\mathrm{m} \mathrm{s}))$

$\eta_{0}-$ regression coefficient $(\mathrm{kg} /(\mathrm{m} \mathrm{s}))$

$f$ - fraction of the active region $(\%)$

$F$ - feed flow rate $(\mathrm{L} / \mathrm{h})$

$F A U-1 \mathrm{FAU}$ is the amount of enzyme that hydrolyzes $5.26 \mathrm{~g}$ starch/h at $30{ }^{\circ} \mathrm{C}$

$F_{\text {evap }}$ - evaporation rate $(\mathrm{L} / \mathrm{h})$

$k_{1}$ - specific branching frequency (tips/(kg DW h))

$k_{2}$ - maximal tip extension rate $(\mathrm{kg} \mathrm{DW} /($ tips $\mathrm{h}))$

$k_{3}$ - rate constant $\left(h^{-1}\right)$

$k_{\text {bran }}$ - specific branching frequency determined by image analysis (tip $/(\mu$ $\mathrm{m} h)$ )

$k_{c}$ - constitutive $\alpha$-amylase production rate (FAU $\mathrm{kg} \mathrm{DW} /(\mathrm{L} \mathrm{g} \mathrm{h})$ )

$K_{c o r}-$ correction constant for the product formation (g glucose/L)

$K_{D O}$ - limit value of dissolved oxygen concentration, below which oxygen

limitation occurs (\%)

$K_{I}$ - Haldane parameter (g glucose/L)

$k_{L} a-$ specific gas-liquid mass transfer coefficient $(1 /(\mathrm{L} \mathrm{h}))$

$K_{S}-$ Haldane parameter (g glucose/L)

$K_{s 1}$ - saturation constant for branching (g glucose /L)

$K_{s 2}$ - saturation constant for tip extension (g glucose /L)

$k_{t i p, \max }-$ maximal tip extension rate determined by image analysis $(\mu \mathrm{m} /$

$(\operatorname{tip} h))$

$m_{o}$ - maintenance coefficient $(\% \mathrm{~kg} \mathrm{DW} /(\mathrm{g} \mathrm{h}))$

$m_{s}$ - maintenance coefficient ( $\mathrm{kg}$ DW g glucose $/(\mathrm{g} \mathrm{L} \mathrm{h})$ )

$\mu_{0}$ - Haldane parameter (FAU kg DW/(L g h))

OUR - Oxygen Uptake Rate ( $\mathrm{mmol} /(\mathrm{L} \mathrm{h}))$

$p-\alpha$-amylase concentration (FAU / L)

$p_{\text {ref }}$ - reference pressure (atm) 
$q_{1}$ - rate of branching $(\mathrm{g} /(\mathrm{kg} \mathrm{DW} \mathrm{h}))$

$q_{2}$ - growth rate of the active region $(\mathrm{g} /(\mathrm{kg} \mathrm{DW} \mathrm{h}))$

$q_{3}$ - rate of hyphal cell formation $(\mathrm{g} /(\mathrm{kg} \mathrm{DW} \mathrm{h}))$

$R$ - universal gas constant (L atm/(mmol K))

$r_{D O}$ - oxygen consumption rate $(\mathrm{kg} \mathrm{DW} /(\mathrm{g} \mathrm{h}))$

$r_{p s}$ - specific $\alpha$-amylase formation rate (FAU $\left.\operatorname{kg} \mathrm{DW} /(\mathrm{L} g \mathrm{~h})\right)$

$r_{s}-$ substrate consumption rate (kg DW g glucose /(g L h))

$\rho$ - hyphal density $\left(\mathrm{g} / \mathrm{cm}^{3}\right)$

$s$ - substrate concentration (g glucose / L)

$s_{f}$ - feed substrate concentration (g glucose / L)

$T_{\text {ref }}$ - reference temeprature $(\mathrm{K})$

$V$ - volume (L)

$w$ - hyphal water content $(\mathrm{g} / \mathrm{kg} \mathrm{DW})$

$x_{a}$ - concentration of active region $(\mathrm{g} / \mathrm{kg} \mathrm{DW})$

$x_{e}$ - concentration of extension zone $(\mathrm{g} / \mathrm{kg} \mathrm{DW})$

$x_{h}$ - concentration of hyphal region $(\mathrm{g} / \mathrm{kg} \mathrm{DW})$

$Y_{P O}$ - stoichiometric coefficient for oxygen consumption for product formation (\% L / FAU)

$Y_{X O}$ - stoichiometric coefficient for oxygen consumption for growth $(\% \mathrm{~kg}$ DW / g)

$Y_{P S}$ - yield coefficient for $\alpha$-amylase on substrate (g glucose / FAU)

$Y_{X S}$ - stoichiometric coefficient for the uptake of substrate (g glucose $\mathrm{kg}$ DW / L g) 

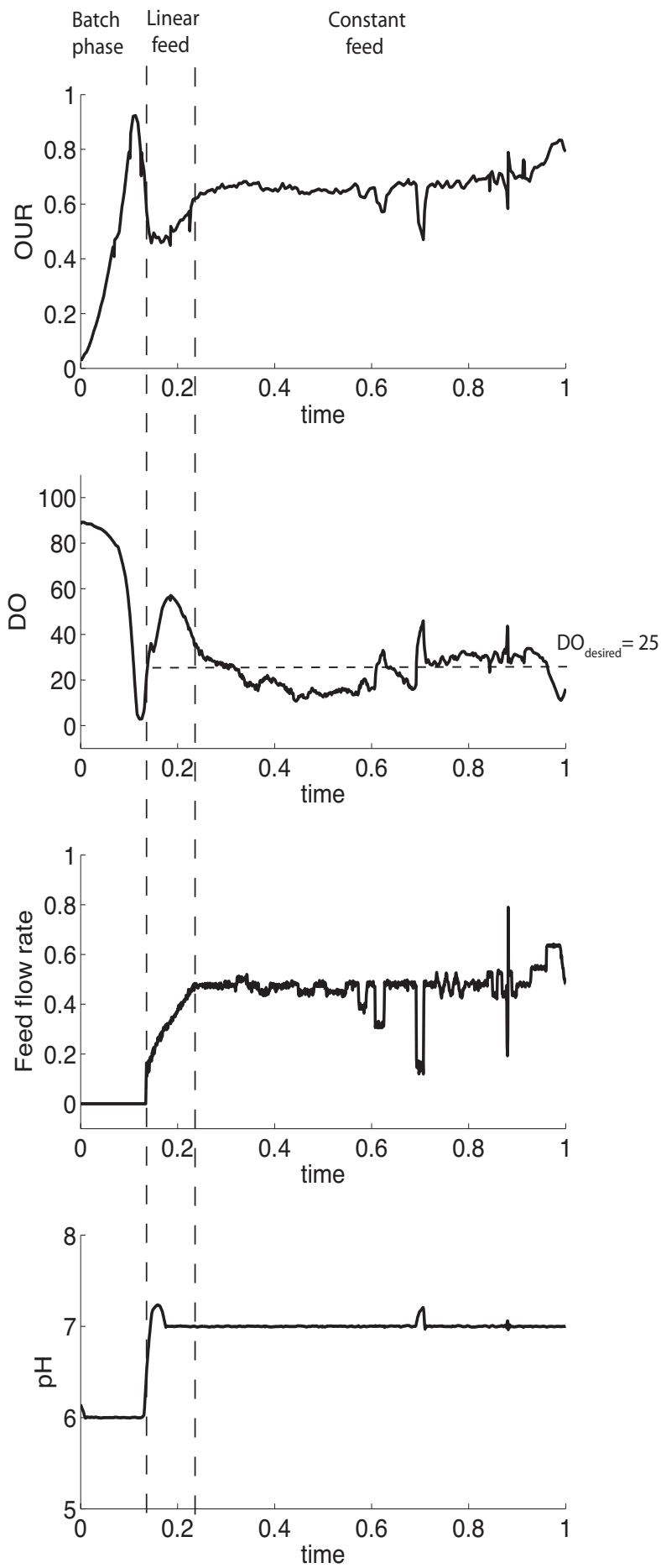

Fig. 1. Experiment I - Current operation in manual mode with three phases (batch operation, linearly-increasing feed, constant feed). 


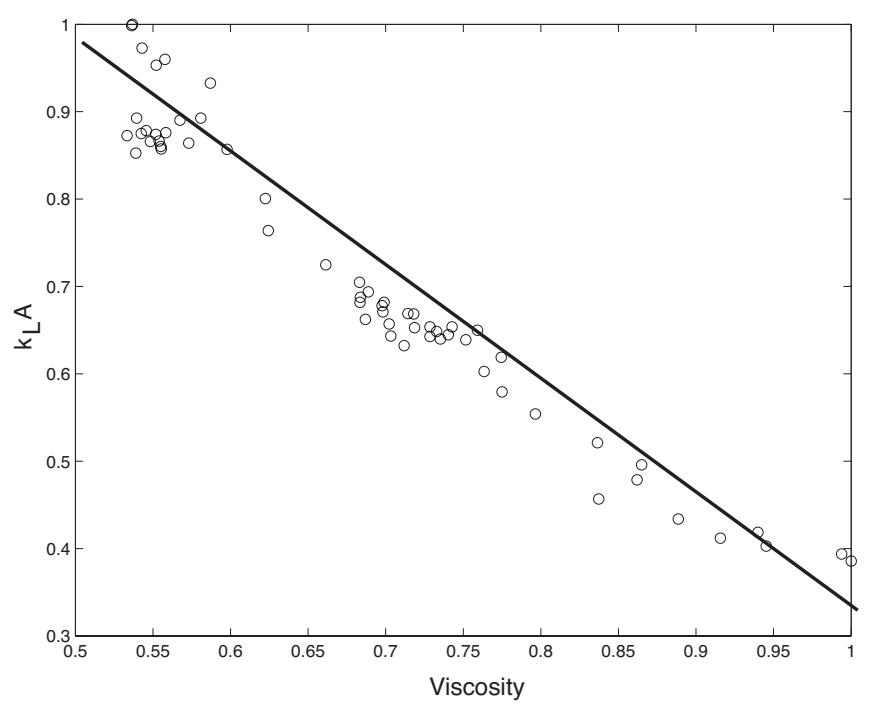

Fig. 2. Relationship between $k_{L} a$ and viscosity during a fed-batch fermentation process. As expected, $k_{L} a$ decreases with increasing viscosity.

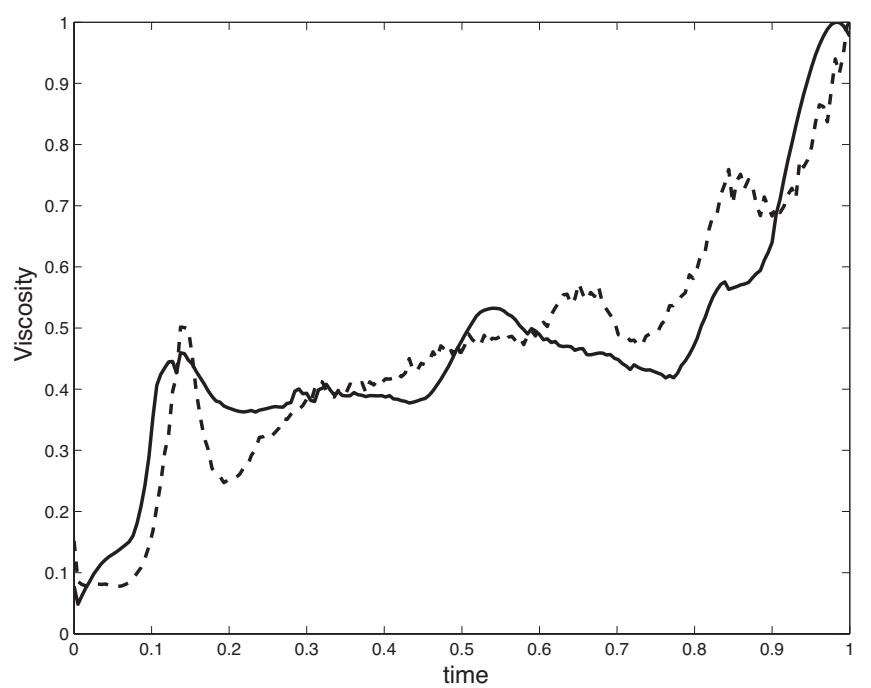

Fig. 3. Evolution of viscosity during fed-batch operation. Measured value (- -) and calculated value using eq. $(24(-))$. 


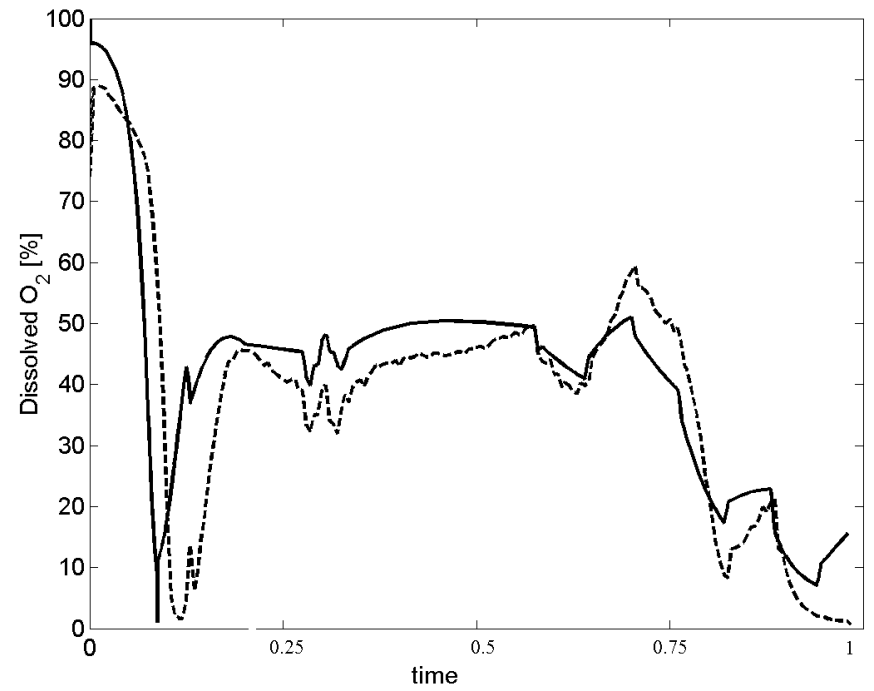

Fig. 4. Dissolved oxygen evolution during fed-batch operation - Data Set 1. Measured (- -) and predicted (-) values as a function of time.

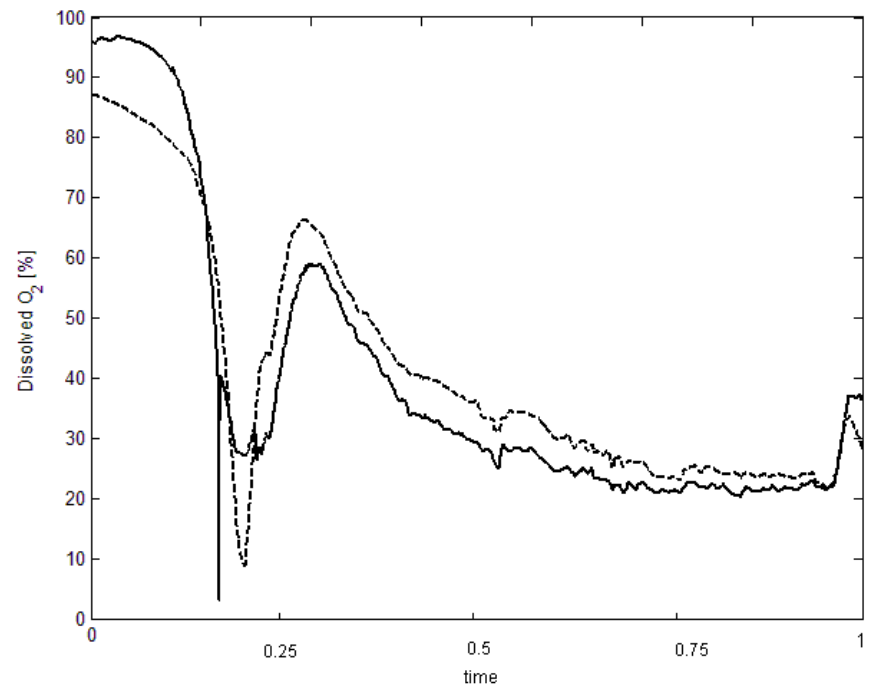

Fig. 5. Dissolved oxygen evolution during fed-batch operation - Data Set 2. Measured (- $)$ and predicted (-) values as a function of time. 

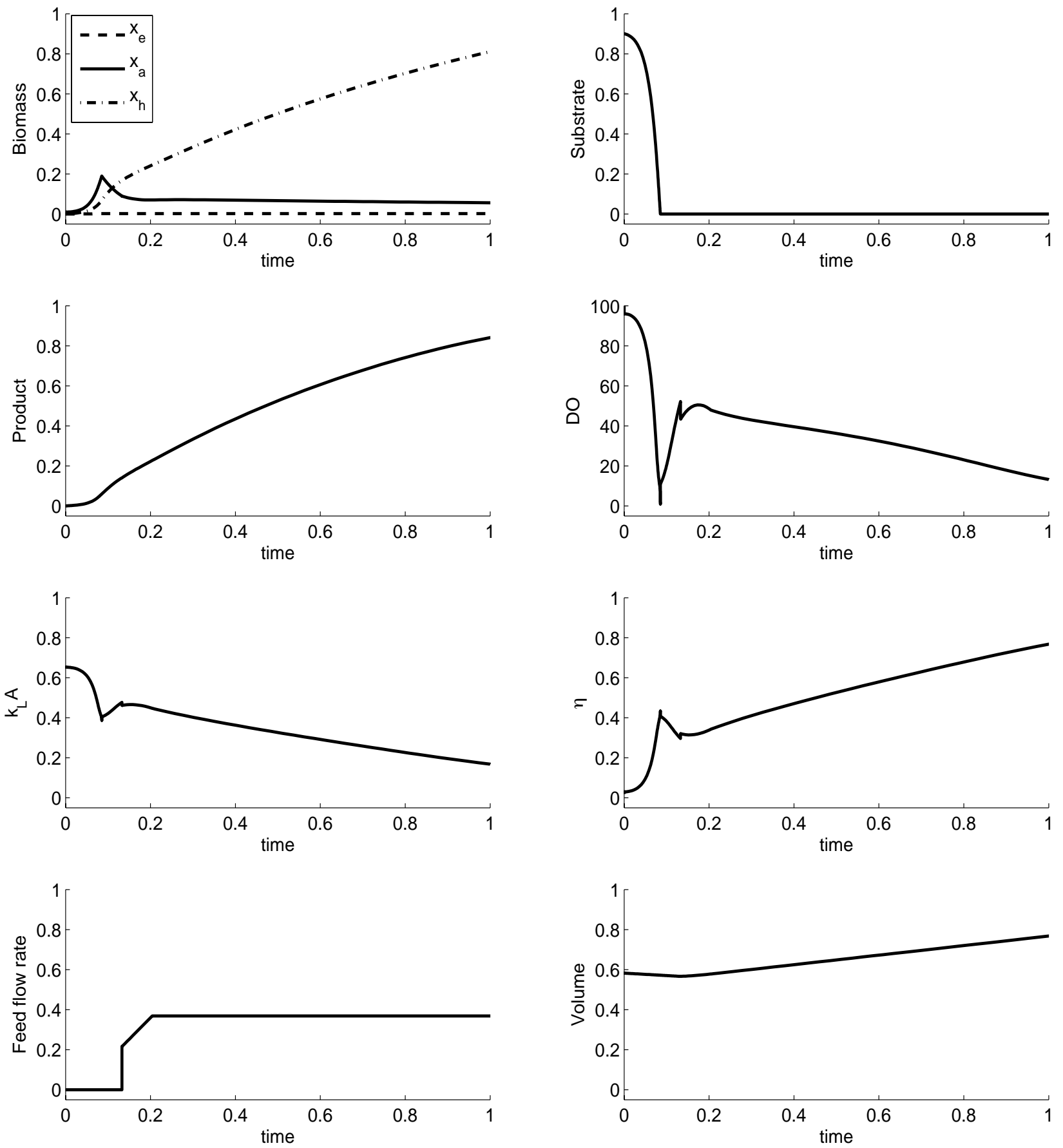

Fig. 6. Simulated states, viscosity and transfer coefficient for an arbitrary feed flow rate. 


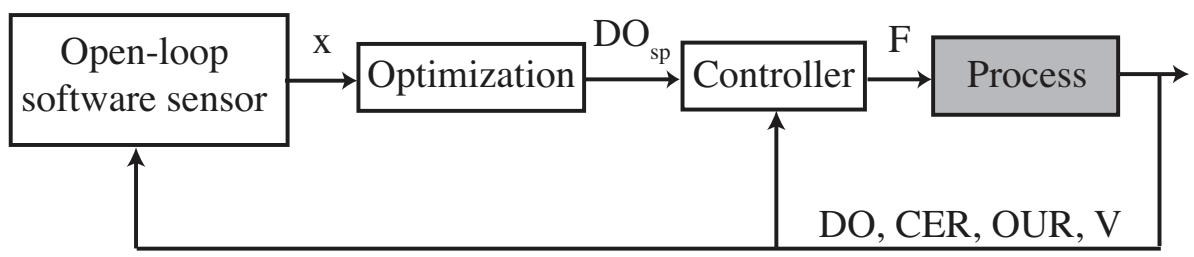

Fig. 7. Computation of $D O_{s p}$ by model-based reoptimization.

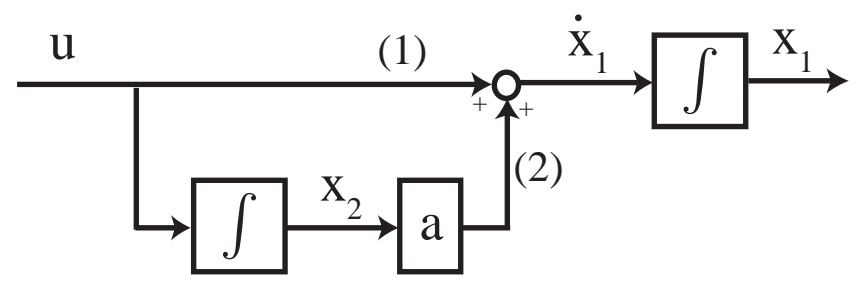

Fig. 8. Illustration of direct (1) and integral (2) effects using a second-order numerical example.

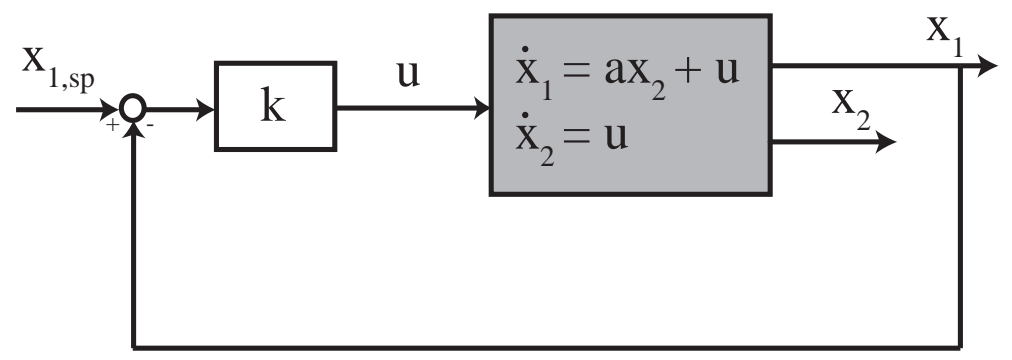

Fig. 9. Output control for the second-order numerical example.

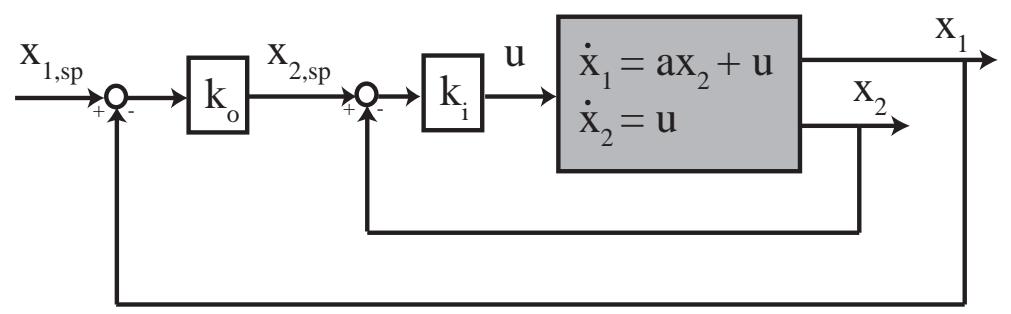

Fig. 10. Cascade control for the second-order numerical example.

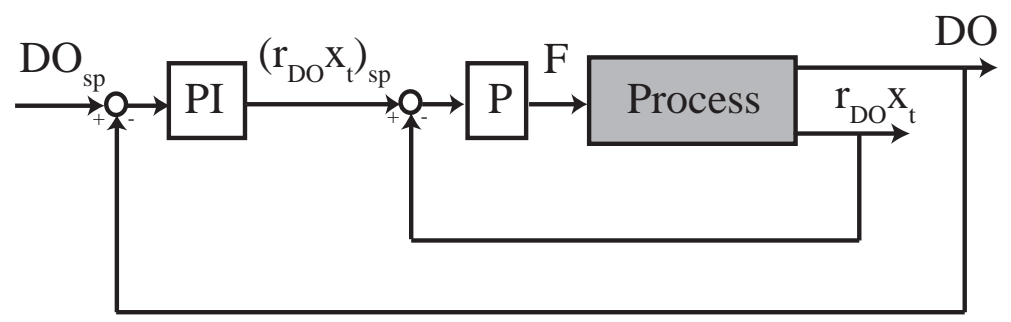

Fig. 11. Cascade control of dissolved oxygen. The oxygen consumption $r_{D O} x_{t}$ is approximated as $\beta \frac{O U R+C E R}{V}$ based on the measurements of OUR, CER and V. 

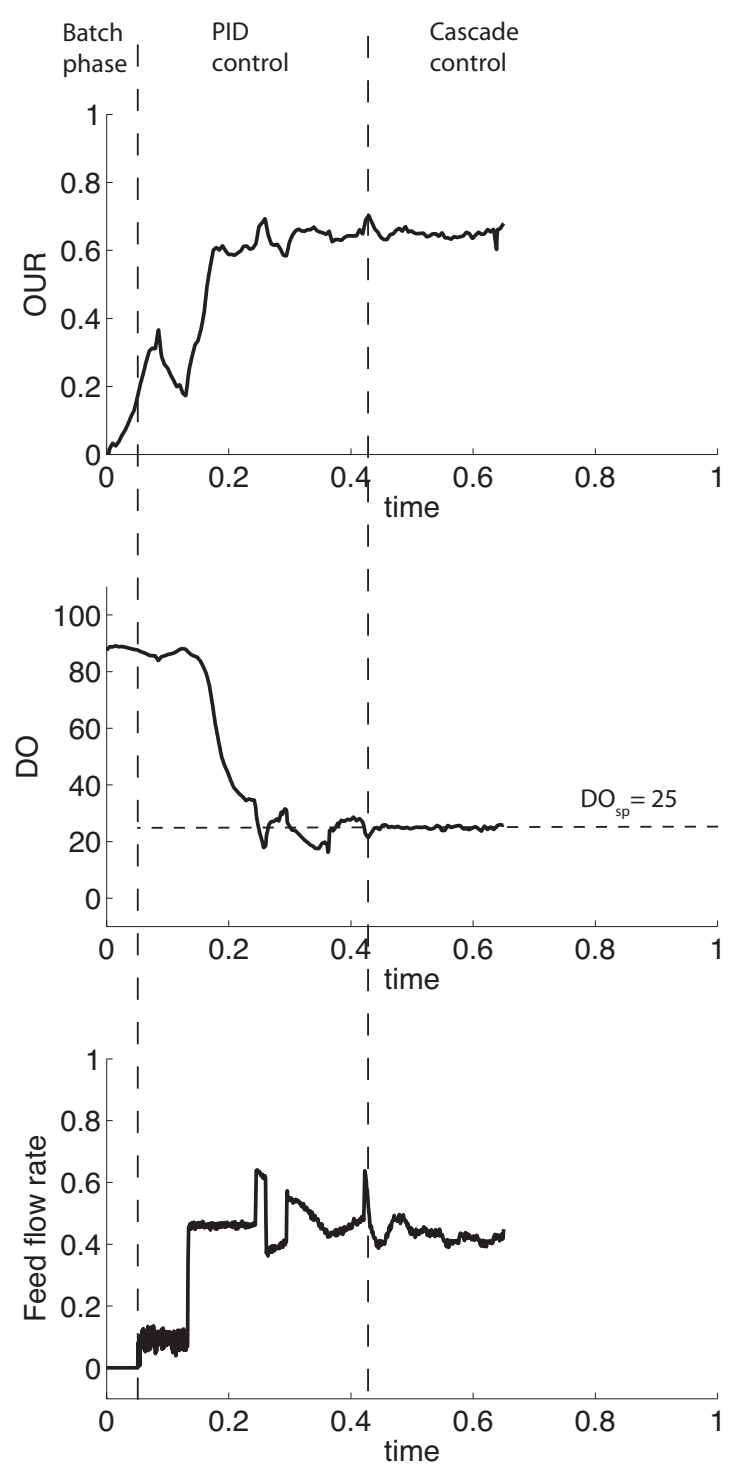

Fig. 12. Experiment II - Batch phase followed by PID control and cascade control. 

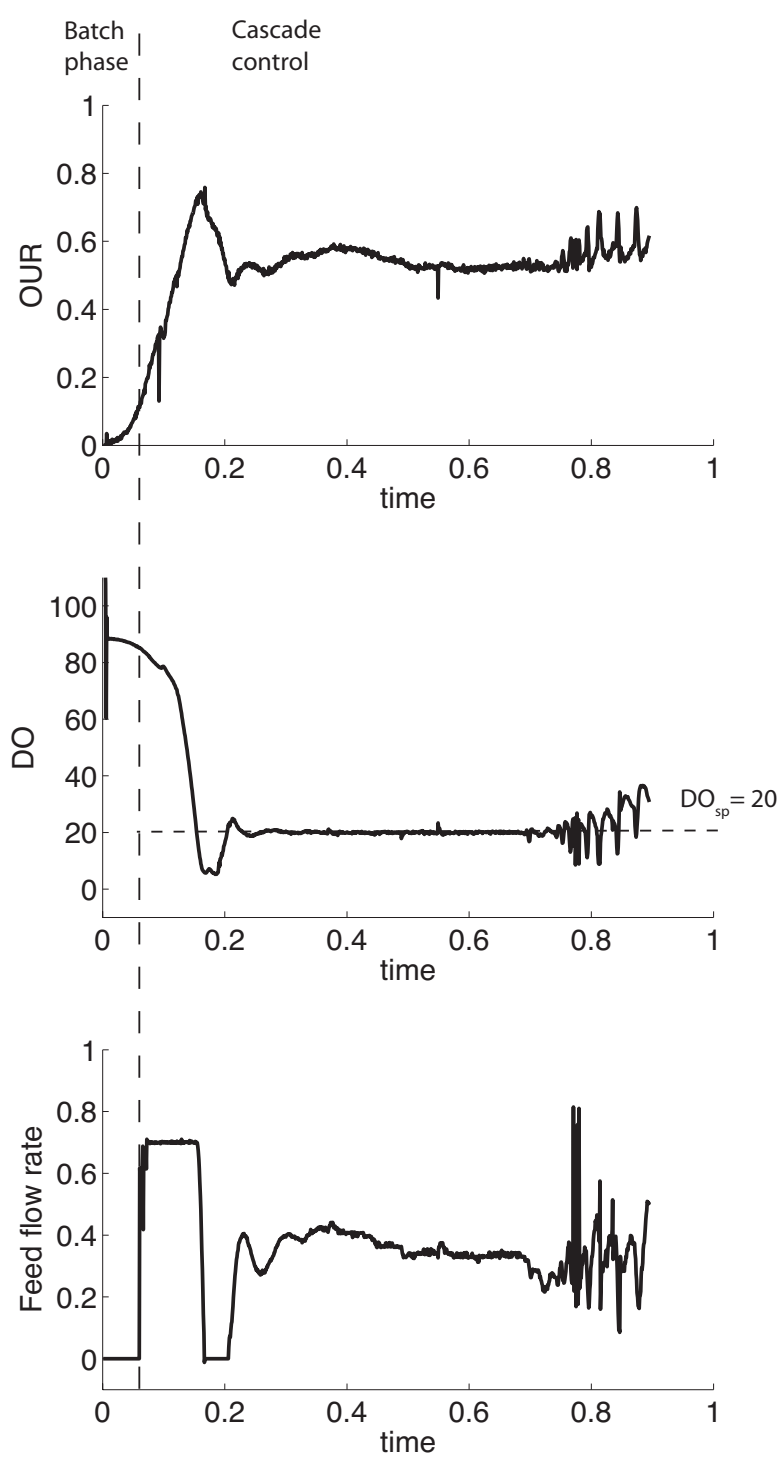

Fig. 13. Experiment III - Batch phase followed by cascade control. The DO setpoint, which has been obtained to optimize the bioreactor performance, is lower than in Experiment II. 


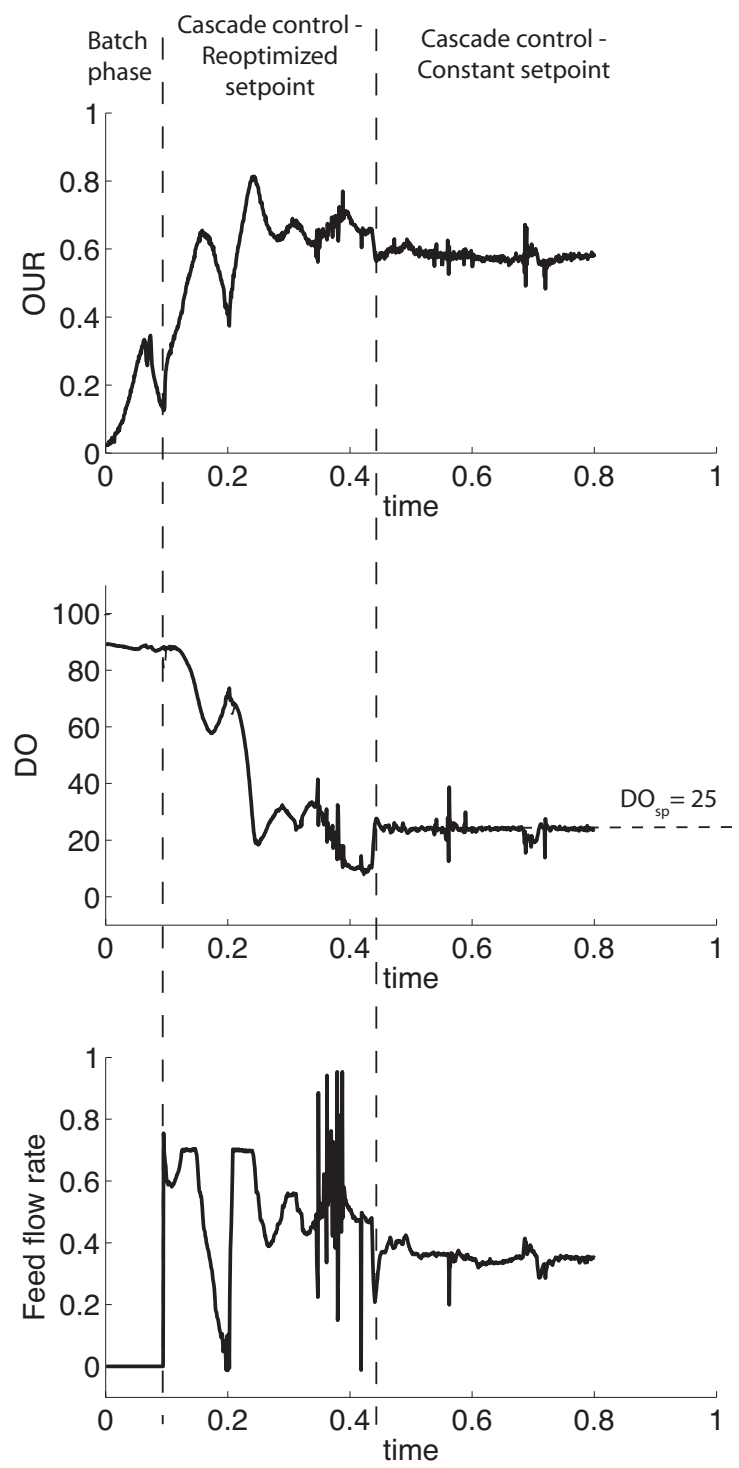

Fig. 14. Experiment IV - Batch phase followed by cascade control using first a reoptimized and then a constant $D O$ setpoint. Note that the curve corresponding to the setpoint is hidden behind the measurement curve. The perturbations in the third phase are due to sensor failure and attempted manual setpoint changes. 